\title{
Properties of photoionized gas in accretion-powered sources
}

\author{
M. Nakayama and K. Masai \\ Department of Physics, Tokyo Metropolitan University, Hachioji, Tokyo 192-0397, Japan
}

Received 13 December 2000 / Accepted 28 May 2001

\begin{abstract}
The UV/X-ray irradiated gas around a compact object was studied by solving the hydrostatic balance coupling with the ionization and thermal balances to obtain the structure over the accretion disk. The gas is ionized and heated by the strong radiation from the compact source, and becomes a recombining plasma; the kinetic energy of the particles is much lower than the energy of the ionization state. The characteristic thermal properties significantly affect the cooling rate, and consequently the structure of the gas. Under gravitation due to the compact object, the structure of the gas is classified into three distinct regimes: (I) a dynamically stable region of high temperature, (II) a stable region of low temperature, and (III) an unstable two-phase region of intermediate temperature. We found that the stability condition $\mathrm{d} T / \mathrm{d} \Xi>0$ does not simply apply to the photoionized gas under gravity, where $T$ is the gas temperature and $\Xi$ is the ratio of the radiation pressure to the gas pressure. Regions I and II are located in that order from the central source toward the outer disk, and region III forms at the interface between the two stable regions. The two phases of high and low temperatures in region III could be smeared marginally by electron conduction, but the dynamically unstable part likely remains at the temperature of $k T \sim 100 \mathrm{eV}$. This characteristic temperature appears purely due to atomic processes and is independent of the source parameters such as the mass or the luminosity of the system. This temperature is of critical importance to the ionization state and radiation mechanisms in the gas. The line-like narrow recombination continua and recombination-cascade lines dominate in the region below $\sim 100 \mathrm{eV}$, while the featureless free-free continuum dominates the radiation spectrum in the region of higher temperatures. These properties are widely true of accretion-powered sources, from stellar-mass systems like X-ray binaries to massive systems like active galactic nuclei, and should be taken into account for the gas dynamics of the accretion flows.
\end{abstract}

Key words. accretion, accretion disks - instabilities - radiation mechanisms: non-thermal - stars binaries: close galaxies: active

\section{Introduction}

In accretion-powered X-ray sources, such as X-ray binaries and active galactic nuclei (AGNs), the gas surrounding a compact source must be strongly irradiated with ionizing photons in the ultraviolet (UV) and X-ray wavelengths. The gas is highly photoionized, but its temperature is relatively low. Thus, the gas becomes strongly recombining and its radiation properties are very different from those of collisionally ionized hot gas, such as ionizing gas in young supernova remnants and quasi-equilibrium gas in clusters of galaxies. Unlike the latter hot gas, in photoionized gas, radiation processes due to excitation by electron-impact are much suppressed and give way to the processes due to recombination capture of slow free electrons into excited states. This drastically affects the cooling rate of the gas; the radiation loss rate is no longer a simple function

Send offprint requests to: K. Masai, e-mail: masai@phys.metro-u.ac.jp of temperature, and the gas should have a complicated structure under strong gravitation by the compact object.

This sort of study on the thermal properties of photoionized gas has been done by several authors: Krolik et al. (1981) on a model of quasar emission line regions, Krolik \& Kriss (1995) and Reynolds \& Fabian (1995) on the warm absorbers of AGNs, Woods et al. (1996) on the coronae and winds from accretion disks, and Hess et al. (1997) from an aspect of the thermal instability in accretion flows of X-ray binaries and AGNs. Also, the related atomic processes have been studied in detail by Hess et al. (1997), Liedahl \& Paerels (1996), and by Savin et al. (1999). The latter two papers address narrow radiative recombination continua and the effect of dielectronic recombination, respectively.

In the present paper, we intend to integrate the detailed atomic and radiation processes into a hydrodynamic balance of the photoionized gas around a compact object. A similar idea has been developed recently by 
Nayakshin et al. (2000) for an accretion disk model where the gas density is determined self-consistently from the hydrostatic balance. Our attention is, however, restricted to the possible atmospheric gas over an existing accretion disk rather than the accretion flow itself, because hydrostatic balance is thought insufficient to evaluate the pressure of accreting gas. We investigated the vertical structure and the stability of the gas under gravitation due to the compact object. One of our motivations is the origin of emission lines of highly ionized metal species. A possible origin is attributed to stellar winds from early-type companion stars in wind-fed systems (e.g. Sako et al. 2000). In disk-fed systems like low-mass X-ray binaries (LMXBs), however, the lines of highly ionized heavy elements could come from a region close to the compact source (Masai et al. 1988), and the related radiation processes have been investigated (Hirano et al. 1987; Raymond 1993). The emission region is stable or likely quasi-hydrostatic, bound gravitationally against the pressure affected by irradiation, over the accretion disk; the gas is not spherically symmetric. Modelling such a disk-fed system, we have carried out the calculations of the thermal and geometrical structures of the gas consistently.

We also examine the ionization structure and the spectral emissivity of the photoionized gas in view of the spectral formation in the warm absorber of AGNs and to the resultant energy loss by radiation out of the gas, in order to investigate the hydrostatic structure. The cooling rate is very sensitive to ionic states as well as to temperature, since the dominant radiation processes are those with which atomic bound states are involved, and is not expressed by a familiar function of the temperature. Therefore, we calculate every process of ionization and radiation at every iteration to obtain a solution of a set of equations.

In the following section, we describe our model calculations. The results and discussion are presented in Sect. 3 . In the final section, we summerize our results and discuss future work. Although we are modelling a system of stellar compact objects in numerical calculations, the obtained characteristics of the thermal and dynamical structure can apply also to massive systems like AGN with an appropriate scaling. Thus, through this paper, we intend to derive quantities in analytic form as far as possible in order to clarify the dependence of parameters taken into our numerical calculations.

\section{Model and calculation}

We consider the gas over a geometrically thin disk around a compact object; the structure of the disk itself is not dealt with, as mentioned in the last section. The gas is photoionized by the primary radiation from the compact object and/or the innermost part of the disk, but must be optically thin for the secondary photons born in the gas.
This constraint means that line emission of highly ionized heavy elements can escape out of the gas to be observed:

$\tau_{\text {line }} \lesssim 10^{-21} \frac{\rho}{\mu \mathrm{m}} l$,

is required for He-like iron (Fe XXV) resonance line at $6.7 \mathrm{keV}$, for example, where $\rho / \mu \mathrm{m}$ is the number density of the gas particles and $l$ the size of the gas in units of c.g.s. Accordingly, the density of the gas concerned must be $\lesssim 10^{12} \mathrm{~cm}^{-3}$ for the systems of neutron stars and stellar black holes.

The thermal structure of the gas depends on the spectrum as well as the number of ionizing photons. We assume a spectrum in the form

$\frac{\mathrm{d} L}{\mathrm{~d} \nu} \mathrm{d} \nu \propto \nu^{-\beta} \mathrm{e}^{-h \nu / k T_{\mathrm{r}}} \nu \mathrm{d} \nu$

for a point central source, where the index $\beta$ represents the fraction of low energy photons and $k T_{\mathrm{r}}$, e-folding energy of the spectrum, represents the characteristic temperature of radiation. The former and the latter parameters play key roles in the degree of ionization and the temperature, respectively, of the photoionized gas. If whole kinetic energy of in-falling gas onto the accretion disk is converted to the local blackbody radiation, i.e., a radiation-dominated disk, the temperature at a radius $r$ of the disk becomes $T_{\text {disk }} \propto r^{-3 / 4}$. Thus, the luminosity depends on $\mathrm{d} L_{\text {disk }} / \mathrm{d} r \propto r^{-2}$, and only the innermost part of the disk contribute as much as the central compact source. Thus, we assume a point source for the radiation field since the inner radius of the disk is small enough compared to the region of interest in this paper. In such a radiation-dominated disk, $\beta \sim 2 / 3$ toward the UV wavelengths, while $\beta \gtrsim 1$ may be expected in optically thin disks like advection-dominated accretion flows with $T_{\mathrm{r}}$ being a cut-off energy of the power-law spectrum.

The thermal structure of the photoionized gas can be scaled with a parameter (e.g. Tarter et al. 1969)

$\xi \equiv \frac{\mu \mathrm{m} L}{\rho R^{2}}$

which means the ratio of the ionizing flux $\sim L / R^{2}$, at the distance $R$ from the source, to the gas density $\rho / \mu \mathrm{m}$; it should be noted in this paper that we take not the hydrogen density but the particle density of the gas to evaluate $\xi$. This sort of $\xi$ parameter is useful if the local cooling time $t_{\text {cool }} \propto T / \Lambda$ is shorter than the sound crossing time $l / c_{\mathrm{s}}$, where $T$ is the gas temperature, $\Lambda$ is the radiative cooling rate per density, and $c_{\mathrm{s}}$ is the sound speed. Our calculations, however, show $t_{\text {cool }}>l / c_{\mathrm{s}}$ for almost the whole region of the gas. The equipartition time between electrons and ions (protons) through Coulomb collisions,

$t_{\mathrm{ei}} \sim 6 \times 10^{-3}\left(\frac{k T}{100 \mathrm{eV}}\right)^{3 / 2}\left(\frac{\rho / \mu \mathrm{m}}{10^{12} \mathrm{~cm}^{-3}}\right)^{-1} \mathrm{~s}$,

is much shorter than $l / c_{\mathrm{s}}$ and $t_{\text {cool }}$. Therefore, the gas should have a pressure-scale structure rather than a 
density-scale one, and $\xi$ is no longer a good parameter. Instead, introducing another parameter (e.g. Krolik et al. 1981)

$\Xi \equiv \frac{L}{4 \pi c R^{2} P}=\frac{\xi}{4 \pi c k T}$,

which is the ratio of the radiation pressure to the gas pressure $P=\rho k T / \mu \mathrm{m}$, we write the hydrostatic equation for the gas as

$$
\frac{\partial \ln P}{\partial h}=-\left(\frac{G M \mu \mathrm{m}}{R^{2} k T}-n_{\mathrm{e}} \sigma_{T} \Xi\right) \frac{h}{R} .
$$

Here $\sigma_{\mathrm{T}}$ is the Thomson scattering cross section, $n_{\mathrm{e}}$ is the electron density, $h$ is the height from the disk plane with $R=\left(r^{2}+h^{2}\right)^{1 / 2}$ at a disk radius $r$, and the gravitational force by the compact object of mass $M$ is taken into account. The self-gravity of the gas and the contribution of the disk mass, $M_{\text {disk }} \lesssim 10^{-8} M_{\odot}\left(\dot{M} / 10^{-8} M_{\odot} \mathrm{yr}^{-1}\right)$ in Xray binaries for example, are negligible as compared with the compact object, where $\dot{M}$ is the mass accretion rate.

We solved Eq. (6) coupled with the ionization - recombination and heating - cooling equations by iteration. The basic scheme follows our previous work (Masai 1984; Hirano et al. 1987; Masai et al. 1988). For abundant species such as $\mathrm{H}$ and $\mathrm{He}$, photoionization is to be coupled with the radiative transfer, as

$$
\begin{array}{r}
\frac{1}{4 \pi R^{2}} \int_{\epsilon_{i j}} \frac{\mathrm{d} \tau_{i j}}{\mathrm{~d} R} \mathrm{e}^{-\tau_{i j}} \frac{\mathrm{d} L}{\mathrm{~d} \epsilon} \frac{\mathrm{d} \epsilon}{\epsilon}+\int_{\epsilon_{i j}} 4 \pi j_{\epsilon} \frac{\mathrm{d} \epsilon}{\epsilon} \\
+n_{\mathrm{e}} S_{i j}(T) n_{i j} \approx n_{\mathrm{e}} \alpha_{i j+1}(T) n_{i j+1},
\end{array}
$$

where

$$
\tau_{i j}(\epsilon)=\int n_{i j} \sigma_{i j}(\epsilon) \mathrm{d} R
$$

is the optical thickness due to photoionization. Here, $n_{i j}$ represents the number density of a species designated by the atomic number $i$ and the charge state $j . \sigma_{i j}$ is the photoionization cross section and $\epsilon_{i j}$ the ionization potential of the species. $\epsilon=h \nu$ the photon energy and $j_{\epsilon}$ represents the spectral volume emissivity of the secondary photons born in the gas. $S_{i j}$ and $\alpha_{i j}$ are the collisional ionization and recombination rate coefficients, respectively. We take $\sigma_{i j} \propto \epsilon^{-3}$ to simplify the argument here. As shown by Masai (1984), a Strömgren sphere solution is derived from Eq. (7) for the boundary condition $\tau\left(\epsilon_{i j}\right)>\tau\left(k T_{\mathrm{r}}\right) \approx$ $\tau\left(\epsilon_{i j}\right)\left(\epsilon_{i j} / k T_{\mathrm{r}}\right)^{3} \gg 1$ with $\epsilon_{i j} / k T_{\mathrm{r}}>1$. This is the case of an $\mathrm{H}$ II region which forms by UV photons around an early type star of $T_{\mathrm{r}} \sim 10^{4} \mathrm{~K}$. However, the above boundary condition no longer applies to photoionization by X-rays, for which $k T_{\mathrm{r}} \gtrsim \mathrm{keV}$ and is much higher than $\epsilon_{i j}$ of the abundant elements. With $\tau\left(\epsilon_{i j}\right) \gg 1$ and yet $\tau\left(k T_{\mathrm{r}}\right) \approx \tau\left(\epsilon_{i j}\right)\left(\epsilon_{i j} / k T_{\mathrm{r}}\right)^{3}<1$, Masai (1984) derived another solution for a sphere ionized by X-ray photons; the radius of the He III region is obtained as

$$
\begin{aligned}
R_{\mathrm{He}} & \approx 1.3 \times 10^{12} \delta^{-1 / 5}\left(\frac{\rho / \mu \mathrm{m}}{10^{12} \mathrm{~cm}^{-3}}\right)^{-3 / 5} \\
& \left(\frac{L}{10^{38} \mathrm{erg} \mathrm{s}^{-1}}\right)^{2 / 5}\left(\frac{k T_{\mathrm{r}}}{2 \mathrm{keV}}\right)^{-2 / 5} \mathrm{~cm}
\end{aligned}
$$

where $\delta$ is a numerical factor of the order of unity depending on the geometrical thickness of the ionization front. It should be noticed that this sphere is somewhat larger with a less sharp ionization front than the Strömgren sphere given formally by $\tau\left(k T_{\mathrm{r}}\right) \gg 1$, i.e., optically thick over the radiation spectrum.

Well within the fully ionized region of $\mathrm{He}$, the equation to be solved reduces to a simple form, because inside there are no more significant opacity sources which deform the primary spectrum. Then, the ionization and recombination balance is determined by

$$
\begin{array}{r}
{\left[\frac{1}{4 \pi R^{2}} \int_{\epsilon_{i j}} \sigma_{i j}(\epsilon) \frac{\mathrm{d} L}{\mathrm{~d} \epsilon} \frac{\mathrm{d} \epsilon}{\epsilon}+n_{\mathrm{e}} S_{i j}(T)\right] n_{i j}} \\
\approx n_{\mathrm{e}} \alpha_{i j+1}(T) n_{i j+1}
\end{array}
$$

for $R<R_{\mathrm{He}}$. The recombination processes include radiative and non-radiative (dielectronic) captures. Practically, the ionization processes are dominated by photoionization, the first term of the left-hand side of Eq. (10), in the conditions concerned here. For the cross section $\sigma_{i j}$ in actual calculations, we used approximated formulae in such respective shells/subshells as $K, L_{\mathrm{I}}, L_{\mathrm{II}+\mathrm{III}}$ and $M$ with corresponding ionization potentials. We take major 15 elements, H, He, C, N, O, F, Ne, Mg, Al, Si, S, Ar, Ca, $\mathrm{Fe}$ and $\mathrm{Ni}$, of astrophysical interest, into account with the Allen (1973) cosmic abundances.

The heating rate per unit volume of the gas due to interaction with photons can be written

$$
\begin{array}{r}
n_{\mathrm{e}} \Gamma=\frac{1}{4 \pi R^{2}}\left[n_{\mathrm{e}} \int \frac{\sigma_{\mathrm{T}} \epsilon}{m_{\mathrm{e}} c^{2}}\left(\epsilon-\frac{21}{5} \frac{\epsilon^{2}}{m_{\mathrm{e}} c^{2}}-4 k T\right) \frac{\mathrm{d} L}{\mathrm{~d} \epsilon} \frac{\mathrm{d} \epsilon}{\epsilon}\right. \\
\left.+\sum_{i j} n_{i j} \int_{\epsilon_{i j}} \sigma_{i j}(\epsilon)\left(\epsilon-\epsilon_{i j}\right) \frac{\mathrm{d} L}{\mathrm{~d} \epsilon} \frac{\mathrm{d} \epsilon}{\epsilon}\right] .
\end{array}
$$

For the local cooling rate per unit volume due to radiation out of the gas, we take into account free-free $(f f)$, freebound $(f b)$ and bound-bound $(b b)$ processes:

$$
\begin{array}{r}
n_{e}^{2} \Lambda=\sum_{i j} n_{e}\left[n_{i j-1} \Lambda_{i j-1}^{b b}+n_{i j}\left(\Lambda_{i j}^{f f}+\Lambda_{i j}^{b b}\right)\right. \\
\left.+n_{i j+1}\left(\Lambda_{i j+1}^{f b}+\Lambda_{i j+1}^{b b}\right)\right],
\end{array}
$$

where the first term in the square bracket represents the loss due to fluorescence lines following innershell ionization, the second is that due to free-free continua, electron-impact excitation lines and two photon decay continua, and the third one is that due to free-bound continua, recombination-cascade lines and dielectronic satellite lines. Actually, the spectral emissivity $\mathrm{d} \Lambda / \mathrm{d} \epsilon$ was calculated at every step and fed back to the cooling rate coefficient $\Lambda$ by integrating over photon energies; that is to say, we did not use a conventional cooling function but calculated self-consistently every step. Because, as demonstrated in Fig. 1, the cooling rate is significantly suppressed under recombining conditions of the photoionized gas, and a conventional function cannot be utilized. 


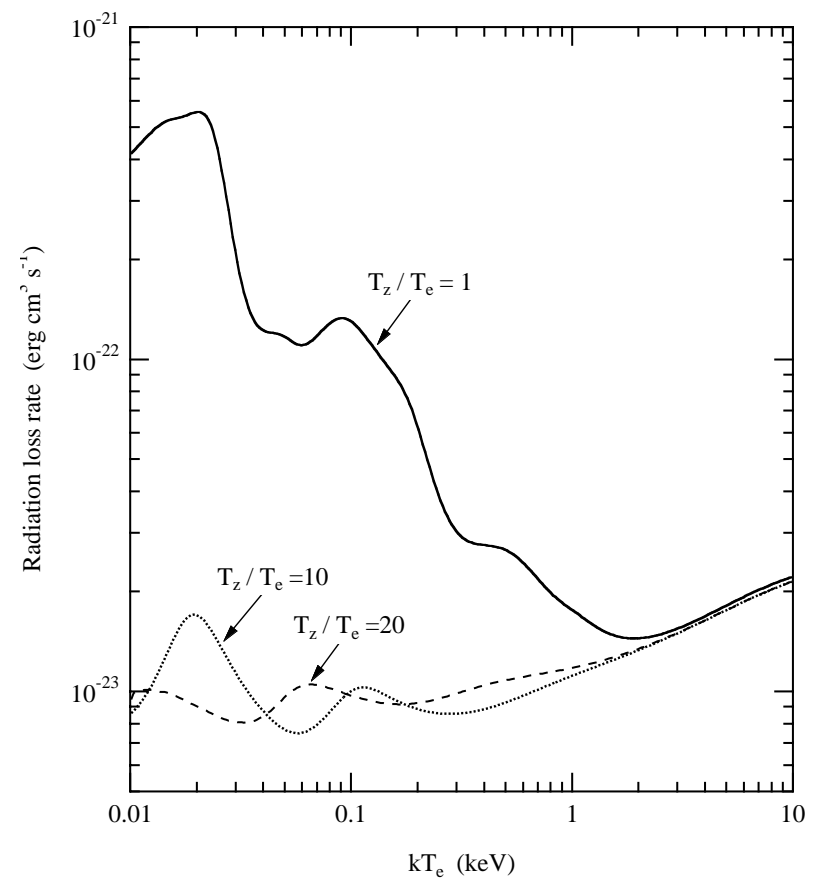

Fig. 1. Radiation loss rate per density as a function of the electron temperature $T_{\mathrm{e}}$ and the ionization temperature $T_{\mathrm{z}}$ (see Masai 1994), where $T_{\mathrm{z}}$ is a measure of ionization state expressed in units of temperature. $T_{\mathrm{z}} / T_{\mathrm{e}}=1$ means that the ionization state is maintained by the kinetic energy of electrons, i.e. collisional ionization equilibrium, and attained in a hot gas such as intracluster medium of clusters of galaxies. A photoionized gas becomes naturally recombining $\left(T_{\mathrm{z}} / T_{\mathrm{e}}>1\right)$, because the kinetic energy of electrons is significantly lower than the energy of ionization state which is maintained by photons. Although $T_{\mathrm{z}} / T_{\mathrm{e}}$ indicates a degree of recombining and is useful in analyzing atomic processes, the actual photoionized gas is not accounted for by a single component thereof.

The longer local cooling time is also in favor of the pressure-scale structure where the cooling time varies $\propto T^{2} / \Lambda$ rather than $\propto T / \Lambda$.

\section{Results and discussion}

\subsection{Density-scale analysis}

Before going into a realistic case of hydrostatic structure, we briefly discuss a structure of density-scale, which is still useful for understanding the local balance in the photoionized gas. The hydrostatic equation can be written similarly to Eq. (6), as

$$
(1-\eta) \frac{\partial \ln \rho}{\partial h}=-\left(\frac{G M \mu \mathrm{m}}{R k T}-2 \eta\right) \frac{h}{R^{2}}
$$

with $\eta \equiv \mathrm{d} \ln T / \mathrm{d} \ln \xi$. An analytic solution is derived approximately to be

$$
\begin{gathered}
\frac{\rho}{\rho_{0}} \approx\left[\frac{\eta}{(1-\eta)(2 \eta-1)} \frac{G M \mu \mathrm{m}}{r k T_{0}}\right. \\
\left.\left\{\left(\frac{R}{r}\right)^{2 \eta-1}-1\right\}+1\right]^{-1 / \eta},
\end{gathered}
$$

where $\rho_{0}=\rho(h=0)$ and $T_{0}=T(h=0)$ are the values at the base of the gas. Depending on the value of $\eta$ which is determined by the local thermal balance, we have three regimes as follows.

(i) $\eta>1$ : At a given $r$ the gas density $\rho$ increases with increasing height $h$. In this region, iron is ionized to Fe XXV and XXVI for $k T_{\mathrm{r}}=2 \mathrm{keV}$ or almost fully ionized for $k T_{\mathrm{r}}=5 \mathrm{keV}$, and could be responsible for its $6.7 \mathrm{keV}$ line. The corresponding ionization parameter is in a range $4.5<\log \xi<5$ for $k T_{\mathrm{r}}=2 \mathrm{keV}$ or $4<\log \xi<4.5$ for $k T_{\mathrm{r}}=5 \mathrm{keV}$. However, no stable hydrostatic structure is realized. (ii) $0<\eta<1$ : The gas has an atmospheric structure where the density decreases with increasing height. Separate two ionization states, $\log \xi<2.5$ and $\log \xi>4.5$, correspond to this regime, and the lower ionization region is responsible for emission lines of various species from $\mathrm{O}$ to $\mathrm{Ca}$. (iii) $\eta<0$ : No hydrostatic solution is obtained. Such a negative value of $\eta$ takes place over the region of $2.5<\log \xi<4$, where the radiation loss rate is affected by radiative recombination edges of highly ionized metals. Such a recombination edge is hidden in a collisionally ionized gas by a much more intense free-free emission continuum, but appears in the photoionized gas where the electron temperature is much lower.

This sort of solution with $\eta<0$ was mentioned by Hatchett et al. (1982; see also Kallman \& McCray 1982). Our calculations, however, show more such regions over a wider range of $\xi$. The difference may be ascribed to the cooling rate due to recombination. We take into account electron capture into atomic levels up to $n=3$ of Li-, Heand H-like ions of all the 15 elements, where $n$ is the principal quantum number. If we took a function smoothing out the recombination edges, we would not have a negative value of $\eta$ but have a smooth curve of $T$ versus $\xi$. Otherwise, if we took into account fewer edges, e.g., captures only into the ground state or a limited number of elements, we would have a smoother curve of $T$ within such an area, as previously reported. This property will be discussed further in the following subsection.

\subsection{Hydrostatic structure}

At gas densities $\lesssim 10^{12}$, as shown in Fig. 2, the local cooling time $t_{\text {cool }} \propto \Lambda / T$ is much longer than the sound crossing time $t_{\mathrm{s}} \sim H / c_{\mathrm{s}}$ in a range of gas temperatures of practical interest, where $H$ is the scale height of the gas. Incorporating the hydrostatic balance, we have a thermal structure with a scale of pressure. With respect to the $T-\Xi$ relation, we can divide the structure of the gas into four regimes characterized by: (I) high $T$ and featureless continuum emission, (II) low $T$ and radiative recombination-cascade lines, (III) medium $T$ and narrow recombination continua, and (IV) no hydrostatic solutions or the gas not bound gravitationally. These regions are located geometrically in the order I, II and IV over the disk from the compact object toward the outer disk, and region III forms around the interface of I 


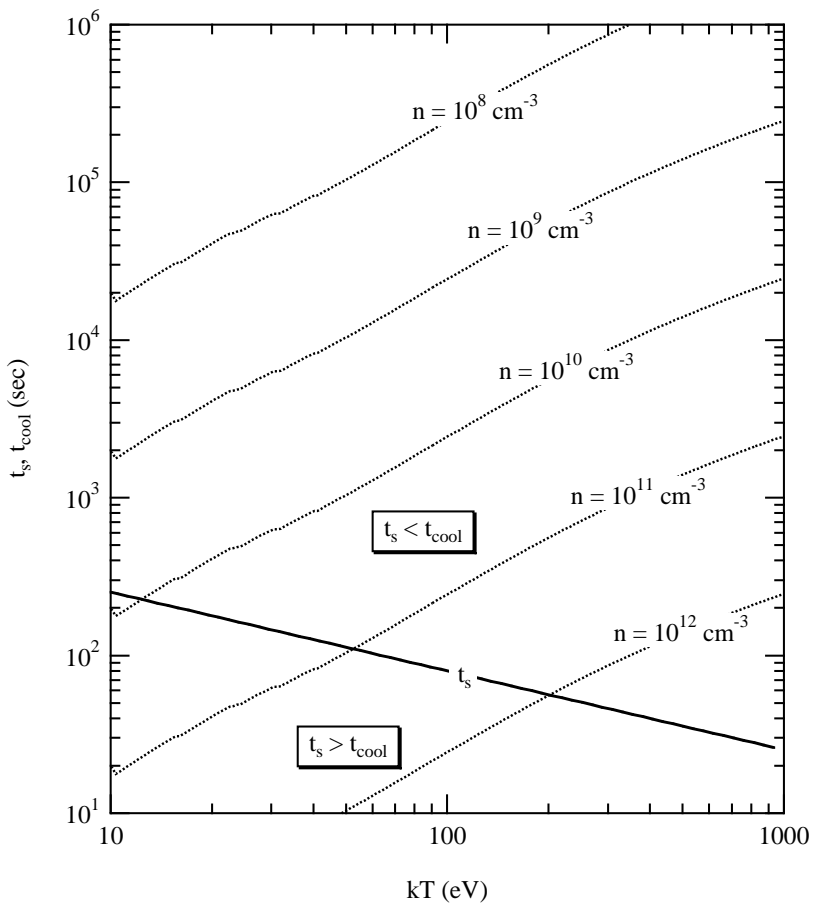

Fig. 2. The local cooling time $t_{\text {cool }}$ is compared with the sound crossing time $t_{\mathrm{s}}=H / c_{\mathrm{s}}$ of the gas; the equipartition between electrons and ions is established in a short time compared to these two time scales. At densities lower than $10^{12} \mathrm{~cm}^{-1}, t_{\mathrm{s}}<$ $t_{\text {cool }}$ over a temperature range of interest. Thus, the gas should have a pressure-scale structure with $\Xi$ over the almost whole region.

and II, as shown in Fig. 3 for a case of $M=1.4 M_{\odot}$, $L=1 \times 10^{36} \mathrm{erg} \mathrm{s}^{-1}, \beta=1$ and $k T_{\mathrm{r}}=2 \mathrm{keV}$, where region $\mathrm{IV}$ is at $r \gtrsim 2 \times 10^{11} \mathrm{~cm}$.

In Fig. 4 we show the thermal structure of the gas in the $T-\Xi$ diagram. We found that $\Xi$ takes its minimum value at $\log \Xi_{\text {crit }} \approx 2.8$. This turning point is determined by the processes that dominate heating and cooling; photoabsorption and free-bound still have an effect on the balance in the $\mathrm{d} T / \mathrm{d} \Xi<0$ regime around $\Xi_{\text {crit }}$, but are giving way to Compton scattering and free-free radiation in the $\mathrm{d} T / \mathrm{d} \Xi>0$ regime. With increasing $T$ from $T\left(\Xi_{\text {crit }}\right), \Xi$ increases monotonically as $\mathrm{d} T / \mathrm{d} \Xi>0$ on the higher $T$ branch. On the other hand, with decreasing $T, \Xi$ increases globally as $\mathrm{d} T / \mathrm{d} \Xi<0$ but walks back and forth locally on the lower $T$ branch. The largest leap appears at $k T \sim 100 \mathrm{eV}$, at which point $\Xi$ takes a local maximum $\Xi_{\mathrm{d}}$. The values such as $\Xi_{\text {crit }}$ and $\Xi_{\mathrm{d}}$ depend on the heating and cooling rates, while the characteristic temperature $\sim 100 \mathrm{eV}$ at $\Xi_{\mathrm{d}}$ is not thereby altered. Region III corresponds to the region between $\Xi_{\text {crit }}$ and $\Xi_{\mathrm{d}}$ in the $T-\Xi$ diagram. In such an irregular structure having a part of $\mathrm{d} T / \mathrm{d} \Xi<0$, the high $T$ and low $T$ phases coexist, and the region concerned may become thermally unstable (e.g., Hess et al. 1997). Also between $\log \Xi_{\mathrm{d}} \approx 3.0$ and $\log \Xi_{\max } \approx 4.4$ the temperature exhibits multi-values, but the high $T$ and low $T$ regimes are geometrically separated from each other. Region II corresponds to the regime of
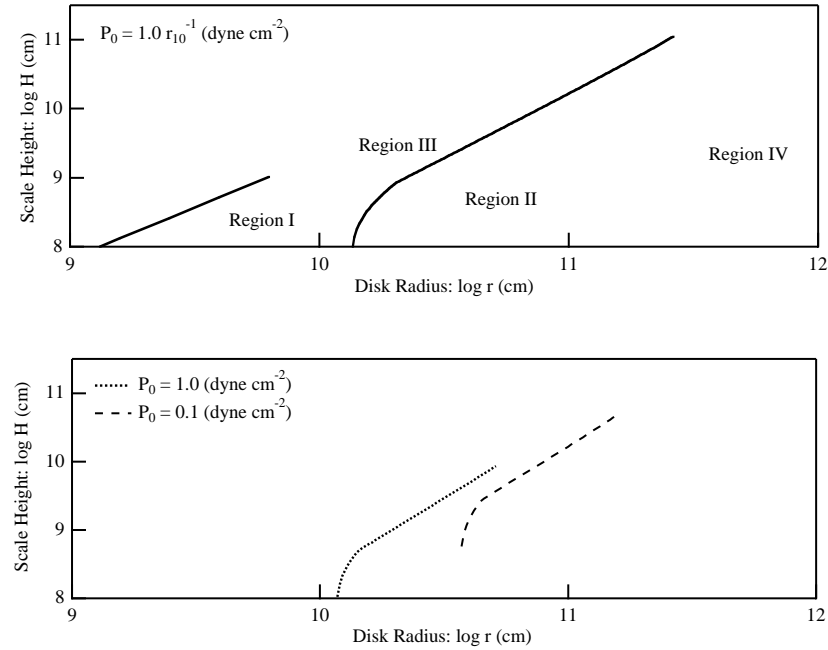

Fig. 3. Hydrostatic structure of the gas: the scale height $H$ is plotted against the disk radius $r$ for the stable regions I and II, and the location of the unstable region III and the gravitationally unbound region IV are also indicated (upper panel). The base pressure is assumed to be $P_{0} \propto r^{-1}$ in the upper panel, while the base pressure is taken to be constant in the lower panel. In the lower panel is shown the dependence on the base pressure of the location of region II where emission lines dominate the spectra.

the low $T$ sequence. Below $k T\left(\Xi_{\max }\right) \sim 10 \mathrm{eV}, \Xi$ decreases again with decreasing $T$ and a branch of $\mathrm{d} T / \mathrm{d} \Xi>0$ appears. However, this regime is located beyond the accretion disk radius of the system considered here.

In region I, every element of the gas is fully ionized, and heating and cooling are balanced through the energy transfer between photons and free electrons by Compton scattering. Then, the gas temperature $T$ approaches an asymptotic value $\sim(1 / 4) T_{\mathrm{r}}$ determined by the spectrum of high energy photons. With the approximation of $T$ being constant, we obtain an analytic solution of Eq. (6) as

$\frac{P}{P_{0}} \approx \exp \left[-\frac{G M \mu \mathrm{m}-L \sigma_{\mathrm{T}} f_{\mathrm{e}} / 4 \pi c}{r k T}\left(1-\frac{r}{R}\right)\right]$,

where $P_{0}=P(h=0)$ is the base pressure and $f_{\mathrm{e}} \equiv$ $n_{\mathrm{e}} \mu \mathrm{m} / \rho$ is the number fraction of electrons. Since $\Xi$ is proportional to the photon pressure $\propto R^{-2}$, this region forms close to the central object. The scale height of the gas is estimated from Eq. (15) to be

$$
\begin{aligned}
H & \sim\left(\frac{2 r^{3} k T}{G M \mu \mathrm{m}-L \sigma_{T} f_{\mathrm{e}} / 4 \pi c}\right)^{1 / 2} \\
& \sim 6.6 \times 10^{7}\left(\frac{r}{10^{9} \mathrm{~cm}}\right)^{3 / 2} \mathrm{~cm},
\end{aligned}
$$

which is a few percent of the radial distance. Although the gas temperature takes its highest value at this region, the gas is bound by the strong gravity of the central object and forms a stable thin atmosphere. Figure 5 shows how $\Xi$, the pressure, the density and the temperature of the gas vary with increasing the height $h$. Region I corresponds to the structure in Fig. 5a, where the gas density is e-folding at $h \sim H$. 

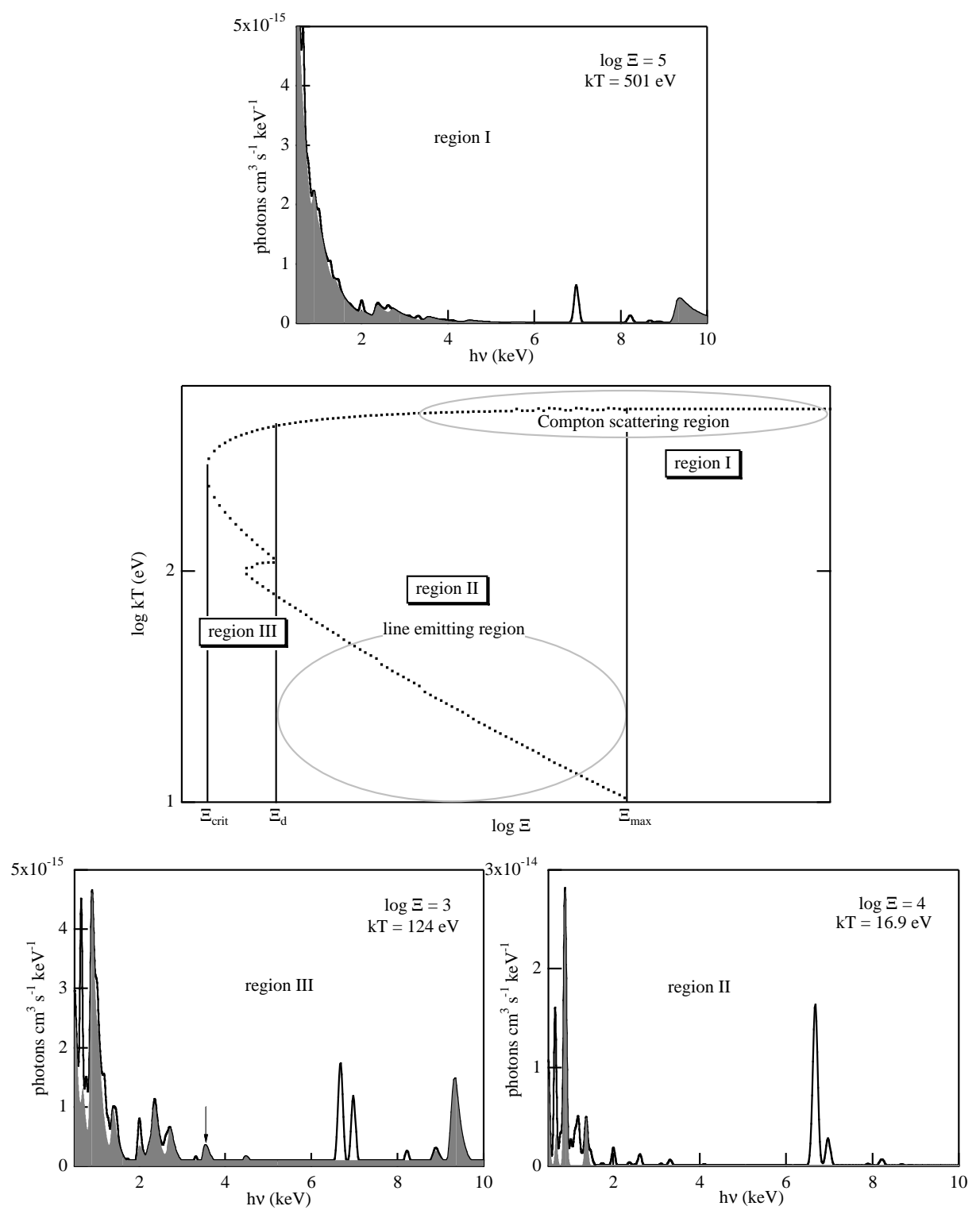

Fig. 4. The gas temperature $T$ versus $\Xi$ (the dotted line in the mid panel) and a typical radiation spectrum of each region. Regions I, II and III correspond to the high $T$ regime in $\Xi>\Xi_{\mathrm{d}}$ (Compton scattering region), the low $T$ regime in $\Xi_{\mathrm{d}}<\Xi<\Xi_{\mathrm{max}}$ (line emitting region) and the regime in $\Xi_{\text {crit }}<\Xi<\Xi_{\mathrm{d}}$, respectively. The radiation spectra are convoluted to a Gaussian kernel with $F W H M=0.07 \epsilon^{0.03} \mathrm{keV}$, where $\epsilon=h \nu$ is the photon energy. The arrow in the region III spectrum indicates the recombination radiation detected by ASCA (see text).

At $\Xi_{\mathrm{d}}<\Xi<\Xi_{\max }$ the gas temperature takes multivalues, largely shaped into two sequences, for a given $\Xi$. One is a high $T$ sequence and the other is a low $T$ sequence, and these two regions are geometrically separated from each other. With $\eta * \equiv \mathrm{d} \ln T / \mathrm{d} \ln \Xi$, an analytic solution of Eq. (6) is obtained approximately in the form

$$
\begin{aligned}
\frac{P}{P_{0}} \approx & {\left[\frac{\eta *}{2 \eta *-1} \frac{G M \mu \mathrm{m}-L \sigma_{\mathrm{T}} f_{\mathrm{e}} / 4 \pi c}{r^{-(2 \eta *-1)} k T_{0}}\right.} \\
& \left.\left(\frac{L}{4 \pi c P_{0}}\right)^{-\eta *}\left\{\left(\frac{R}{r}\right)^{2 \eta *+1}-1\right\}+1\right]^{-1 / \eta *}
\end{aligned}
$$

for both the regions. The region corresponding to the high $T$ sequence is connected to region I in both geomet- rical and thermal senses. On the other hand, the thermal property of the low $T$ sequence is very different from region I. We designate this region of low $T$ as region II. The vertical structure is shown in Fig. 5b, where one can see that the gas also has a finite scale height in region II. The $T$ sequence in region II is not a smooth line but consists of more detailed multi-values. Such a complicated structure comes from the cooling rate due to free-bound and following bound-bound emissions which are very sensitive to the electron temperature and the ionic state of the metal species. One can see the effect of cooling on the structure of region II in Fig. 6, where the relative abundance of iron is varied to demonstrate the effect. A more irregular structure would appear due to other metal 

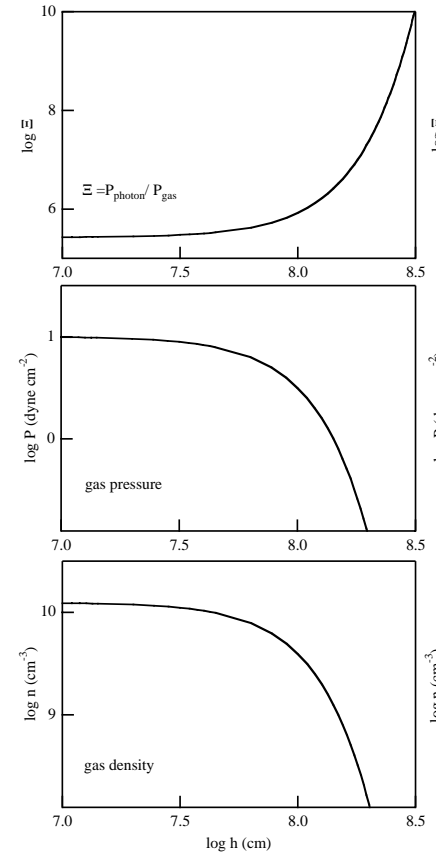

(a)
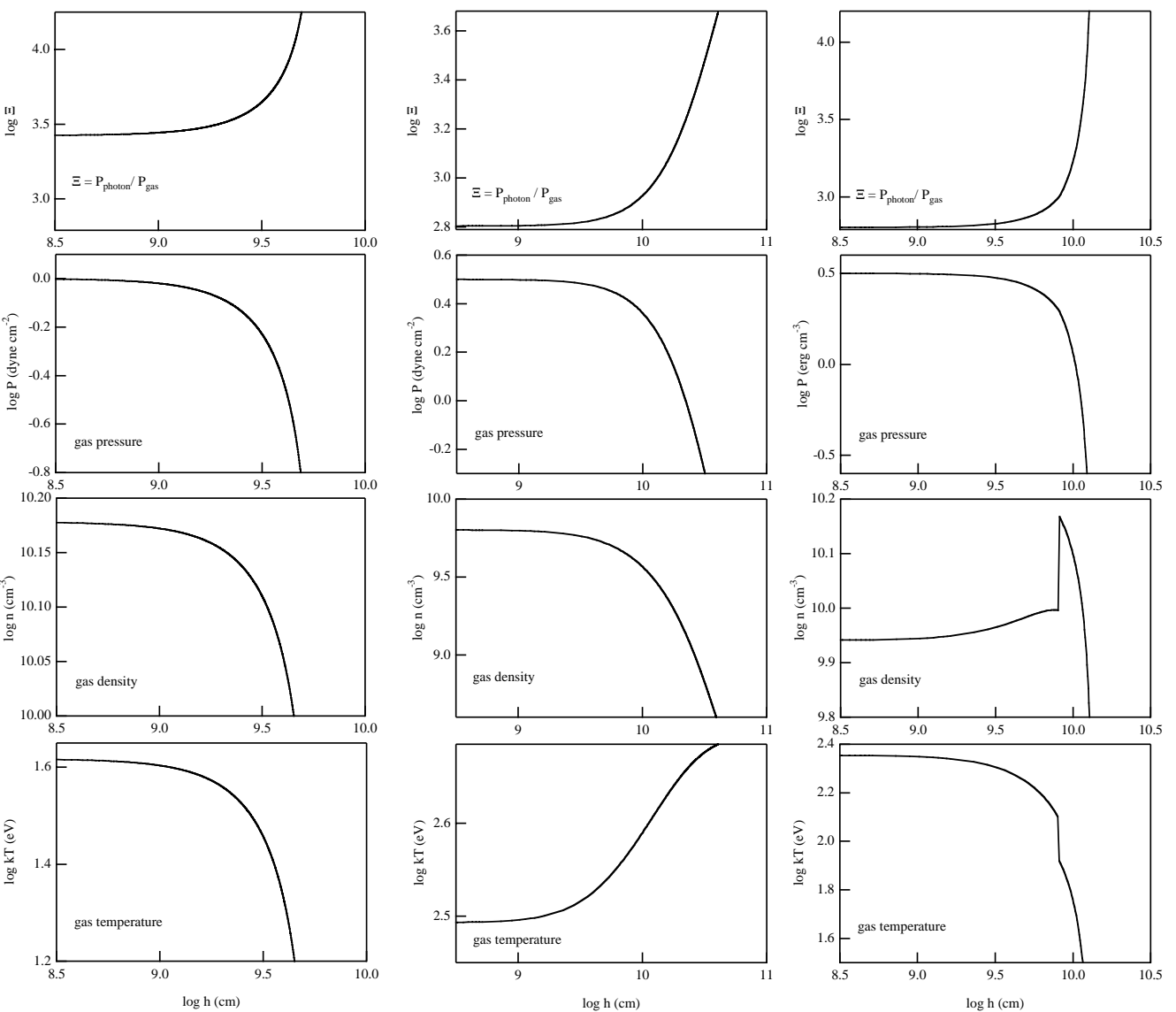

(c)

Fig. 5. The vertical structure of the gas at a disk radius: a) region $\mathrm{I}$ at $\log r(\mathrm{~cm})=9.0, \mathbf{b}) \operatorname{region} \mathrm{II}$ at $\log r(\mathrm{~cm})=10.5$, and c) region III at $\log r(\mathrm{~cm})=10.56$. The gas temperature in region I is omitted because it is almost constant. It is found that regions I and II have a finite scale height, while region III is composed of the two regimes of high (left panel) and low (right panel) temperatures and the latter is subject to dynamical instability.

species if the iron abundance were lower; that is to say, region II is easy to be affected by a cooling model or radiation processes taken into account (see also Hess et al. 1997; Savin et al. 1999). The radiation spectrum of region II is dominated by recombination lines and narrow recombination continua of metal species, in contrast to that of region I which is dominated by the free-free continuum due to abundant elements such as $\mathrm{H}$ and $\mathrm{He}$.

The regime of $\mathrm{d} T / \mathrm{d} \Xi<0$ has been investigated and thought to be thermally unstable (e.g., Krolik et al. 1981). It is true to isobaric perturbations; if $\mathrm{d}\left(\Lambda-\Gamma / n_{\mathrm{e}}\right) / \mathrm{d} T<0$, the gas is thermally unstable and would cool or heat toward a different equilibrium temperature which will be stable. Actually, such a behavior appears also in our calculations of Eq. (13) with the density being a variable, and no finite atmosphere is obtained for $\mathrm{d} T / \mathrm{d} \xi<0$, as mentioned in the previous section. In the hydrostatic calculations of Eq. (6) with the pressure being a variable, the vertical structure of region II, however, exhibits a finite atmosphere in spite of $\mathrm{d} T / \mathrm{d} \Xi<0$. It should be noted that $\mathrm{d} T / \mathrm{d} \Xi$ does not always have the same sign as $\mathrm{d} T / \mathrm{d} \xi$. Instead of the $\mathrm{d} T / \mathrm{d} \Xi$ criterion, we should discuss the dy- namical stability under gravity. The condition for stability to convective motions is

$$
\frac{\mathrm{d} \ln \rho}{\mathrm{d} h}<\frac{1}{\gamma} \frac{\mathrm{d} \ln P}{\mathrm{~d} h}
$$

where $\gamma=[\partial \ln P(1+\Xi) / \partial \ln \rho]_{S} \sim 4 / 3-5 / 3$ is the adiabatic index, i.e., with the entropy $S$ being constant. Equation (18) reduces to $l_{\rho}<\gamma l_{\mathrm{P}}$ for $\mathrm{d} \ln \rho / \mathrm{d} h<0$ and $\mathrm{d} \ln P / \mathrm{d} h<0$, where $l_{\rho}$ and $l_{\mathrm{P}}$ are the density and the pressure scale lengths, respectively. This condition is satisfied in regions I and II, while not satisfied in region III. The low temperature regime of region III is totally unstable, as shown in the right panel of Fig. $5 \mathrm{c}$. Even the high temperature regime of region III is not stable because $l_{\rho} \gtrsim \gamma l_{\mathrm{P}}$, although it exhibits $\mathrm{d} T / \mathrm{d} \Xi>0$ and an apparent finite atmosphere on account of $\mathrm{d} T / \mathrm{d} h>0$ with $\mathrm{d} P / \mathrm{d} h<0$, i.e., the density decreases faster than the temperature increases. Consequently, region II of $\mathrm{d} T / \mathrm{d} \Xi<0$ is found stable, but region III consisting of the two phases is unstable under the gravity present. This sort of stability is not discussed by Nayakshin et al. (2000), who take hydrostatic balance 


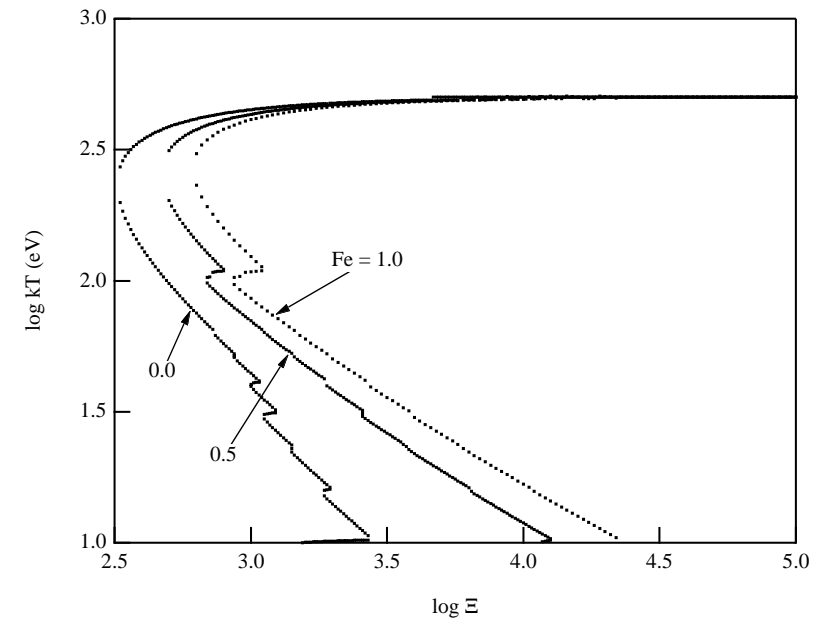

Fig. 6. $T-\Xi$ relation is plotted for the iron abundance, 0.0 , 0.5 and 1.0 (same as Fig. 4) relative to Allen's value, to demonstrate the effect of atomic processes on the thermal structure. It is found that the distinct leap at $\sim 100 \mathrm{eV}$ disappears when the iron abundance becomes zero. Also found is that the low temperature sequence (region II) is influenced easily by the atomic processes.

into account for photoionized accreting gas and investigate the thermal instability based on $\mathrm{d} T / \mathrm{d} \Xi$.

Region III is characterized by a distinct leap in $\Xi$, which appears at around $k T \sim 100 \mathrm{eV}$ due to recombination of the $K$-edge of H-like iron (Fe XXVI). The high $T\left(>T\left(\Xi_{\text {crit }}\right)\right)$ branch of this region is smoothly connected to that in $\Xi_{\mathrm{d}}<\Xi<\Xi_{\max }$ and consequently to region $\mathrm{I}$. On the other hand, in the low $T$ branch, the thermal and radiation properties are similar to those in region II, but the temperature is higher. Then, the recombination radiation falls in a slightly wider range of photon energies than that in region II, while the emissivity of the recombination-cascade lines becomes smaller. Hence, region III is dominated more by narrow recombination continua than lines compared to region II. The geometrical structure still obeys Eq. (17) in region III. The higher temperature part of $\eta *>0$ is smoothly connected to the high $T$ sequence $(\eta *>0)$ of region I, while the low temperature part is connected to region II. Consequently, region III forms at the interface between regions I and II, and consists of two phases of high and low temperatures. In Fig. $5 \mathrm{c}$ are shown the vertical structures of these two phases. It should be noted that region III contains a dynamically unstable part of $T \sim 100 \mathrm{eV}$ in the low temperature phase. Thermal conduction by electrons may diminish the temperature gap and smear the two-phase thermal structure around this region. In terms of $t_{\mathrm{ei}}$, the conduction time scale can be estimated as

$$
\begin{aligned}
t_{\text {cond }} & \sim \max \left[\frac{l_{\mathrm{T}}^{2}}{\kappa}, \frac{l_{\mathrm{T}}}{T^{1 / 2}}\right] \geq \frac{l_{\mathrm{T}}^{2}}{\kappa} \sim\left(\frac{l_{\mathrm{T}}}{\lambda_{\mathrm{e}}}\right)^{2} \frac{m_{\mathrm{e}}}{m_{\mathrm{i}}} t_{\mathrm{ei}} \\
& \sim 3 \times 10^{-4}\left(\frac{l_{\mathrm{T}}}{\lambda_{\mathrm{e}}}\right)^{2}\left(\frac{\rho / \mu \mathrm{m}}{10^{10} \mathrm{~cm}^{-3}}\right)^{-1} \mathrm{~s} .
\end{aligned}
$$

Here $l_{\mathrm{T}} \sim(\nabla \ln T)^{-1}$ is the scale length of the temperature, $\kappa$ the diffusion coefficient, $\lambda_{\mathrm{e}}$ the mean free path of electrons, and the temperature is fixed at $k T \sim 100 \mathrm{eV}$, at which the largest leap appears due to atomic processes, i.e., independent of the geometrical parameters. The function max in Eq. (19) means that the conduction heat flux becomes saturated at $\lambda_{\mathrm{e}} \gtrsim l_{\mathrm{T}}$; whether it is the case or not, $t_{\text {cond }}$ should be longer than its value in the diffusive heat flux. The temperature scale length is estimated as $l_{\mathrm{T}} \sim 6 \times 10^{9} \mathrm{~cm}$ from Figs. 3 and $5 \mathrm{c}$. The mean free path is $\lambda_{\mathrm{e}} \sim 2 \times 10^{6} \mathrm{~cm}$ for a typical density $\rho / \mu \mathrm{m} \sim 10^{10} \mathrm{~cm}^{-3}$ of the unstable region. Then, $l_{\mathrm{T}} / \lambda_{\mathrm{e}} \sim 3 \times 10^{3}$ and consequently $t_{\text {cond }} \sim 3 \times 10^{3} \mathrm{~s}$, which is marginally longer than $t_{\text {cool }}$ shown in Fig. 2. Thus, the large leap in the thermal structure is not smeared by thermal conduction but remains partially in the dynamically unstable part of the geometrical structure.

\subsection{Radiation properties}

In the photoionized gas, radiative recombination is enhanced to play a role in atomic level populations and the resultant thermal emission. The cross section of electron capture into a level of principal quantum number $n$ can be expressed as

$\sigma_{i j, n} \propto \frac{1}{n^{3}}\left(\frac{3}{2} \frac{k T}{\epsilon_{i j}}+\frac{1}{n^{2}}\right)^{-1}$

with the hydrogen approximation $\epsilon_{i j, n}=\epsilon_{i j} / n^{2}$ for the ionization potential of atomic level $n$. In a collisionally ionized gas, the kinetic energy of free electrons maintains the ionization state, i.e., $k T \sim \epsilon_{i j}$. Therefore, $\sigma_{i j, n} \propto n^{-3}$ in the coronal limit, and most of the electrons are captured directly into the ground state $(n=1)$, emitting photons over a broad energy range from $\epsilon_{i j}$ through $\sim \epsilon_{i j}+k T$. However, this is not the case in the photoionized gas. Since the kinetic energies of free electrons are significantly low compared to the ionization potential, $k T \ll \epsilon_{i j}$, the total cross section of capture is reduced as

$$
\begin{aligned}
\sigma_{i j}=\sum_{n} \sigma_{i j, n} \propto \sum_{n} \frac{1}{n} & \left(1+\frac{3}{2} \frac{k T}{\epsilon_{i j, n}}\right)^{-1} \\
& \sim\left[\sum_{n=1}^{n *} \frac{1}{n}\right]+\frac{1}{2},
\end{aligned}
$$

where $n *=\left(2 \epsilon_{i j} / 3 k T\right)^{1 / 2}$ is an effective quantum number at which the depth of the potential well is comparable to the average energy of free electrons. In our calculations, $n * \sim 8$ and the population by electron capture becomes important for atomic levels up to $n=3-4$. Hence, the capture into excited levels increases more than those directly into the ground state, and are followed by cascades to the ground state. The photons emitted during the capture into an excited level fall into a quite narrow energy band of width $\sim k T\left(\ll \epsilon_{i j}\right)$, while the photons emitted through the cascades dominate the line spectra. The narrow recombination continua look like lines (Liedahl \& Paerels 1996), 
and the cascades enhance multiplet lines more than the resonance line (Masai 1994; Porquet \& Dubau 2000).

These are seen in the spectra of regions III and II in Fig. 4, where the continuum is represented by the filled area to be distinguished from the real lines. It is noted that the line center of the He-like iron $K \alpha$-blend ( $n=1-2$ transitions) slightly shifts toward the forbidden line ${ }^{1} \mathrm{~S}-{ }^{3} \mathrm{~S}$ $(6.63 \mathrm{keV})$ and the intercombination line ${ }^{1} \mathrm{~S}-{ }^{3} \mathrm{P}(6.67 \mathrm{keV})$ from the resonance line ${ }^{1} \mathrm{~S}-{ }^{1} \mathrm{P}(6.70 \mathrm{keV})$, compared to a spectrum of collisionally ionized hot gas. The arrow in the region III spectrum indicates the recombination edge detected by the ASCA Cyg X-3 observation (Kawashima \& Kitamoto 1996). Our model predicts more intense narrow recombination continua in the $2-3 \mathrm{keV}$ range covered by ASCA, but those were not claimed by the authors. This is likely because the authors could identify the narrow continuum with a line. In fact, our calculations show that lines exist so close to each continuum edge in $2-3 \mathrm{keV}$ as to be blended into a line-like shape with the resolving power of ASCA. On the other hand, the detected $\sim 3.5 \mathrm{keV}$ edge (and also the faint $\sim 4.5 \mathrm{keV}$ edge) is free of neighbouring intense lines. Also around $1 \mathrm{keV}$, we predict line-like recombination continua blended with many lines. The width of the recombination continuum is narrower in region II than in region III because the temperature is lower in region II. The emission lines are more intense in region II than in region III. From this result, it is also inferred that the line emission is dominated by radiative recombinationcascade processes, which are further enhanced by decreasing the temperature in the recombining gas (see Fig. 6 in Masai 1994).

\section{Concluding remarks}

We summarize the results of the present work and discuss their application to more general cases:

1. In accretion-powered sources, the gas surrounding a compact object is strongly irradiated by ionizing photons and exhibits characteristic properties, which affect the cooling rate of the gas and the dynamics of the accretion flow. We investigated the hydrostatic structure of such a gas over the geometrically thin disk. Since the sound crossing time in the scale height of the gas is shorter than the local cooling time, a pressurescale structure is preferred under gravitation due to the central compact object.

2. The innermost part (region I) of the gas is bound by gravitation with a finite scale height. The gas is heated by strong irradiation to become as hot as the primary photons from the source, i.e., close to the radiation temperature. Every element is fully ionized, and the gas emits featureless free-free continuum radiation.

3 . Outside the hot gas region I, there exists another stable region (region II) of lower temperatures $(<100 \mathrm{eV})$, bound by gravitation. Heavy elements are highly ionized resulting in the radiation of narrow recombination continua and cascade lines. If the persistent spectra of these processes are observed, the emission likely comes from the stable region II. Accordingly, the temperature of the emission region is predicted to be lower than $\sim 100 \mathrm{eV}$.

4. In the interface between the two stable regions, there exists a dynamically unstable region (region III) of intermediate temperature of the order of $100 \mathrm{eV}$, though the gas is partially bound by gravitation. The gas is also thermally unstable and two phases of higher and lower temperatures coexist. Thermal conduction can marginally smear the two phases, but the gas still remains dynamically unstable. Heavy elements are almost fully ionized, and narrow recombination continua dominate the radiation spectra of the gas.

5. The stability criterion of the photoionized gas, $\mathrm{d} T / \mathrm{d} \Xi>0$, holds true for isobaric perturbations. However, this is not simply applicable to hydrostatic balance under gravity. It is found that the gas can be dynamically unstable even for $\mathrm{d} T / \mathrm{d} \Xi>0$ or stable for $\mathrm{d} T / \mathrm{d} \Xi<0$ depending on the radiation spectrum and the mass of a central source and the cooling rate.

6. In a stellar mass system, numerically modelled here, the outermost part of the gas, the stable region of lower temperatures (region II), is terminated by the domain of no solution, i.e., where the gas is no longer gravitationally bound.

7. The thermal properties described in terms of $\Xi$ applies to accretion flows in AGNs, as well as X-ray binaries, by scaling primarily the source luminosity $L$. Scaling the characteristic radiation temperature $T_{\mathrm{r}}$ gives the maximum temperature of the gas heated by irradiation, i.e., the temperature attained in the innermost region I, but it little affects the characteristics of region II and the low $T$ branch of region III.

8. Although the $T-\Xi$ relation is applicable to a wide range of parameters with $\Xi$ scaling, the behavior on the low $T$ branch is influenced by atomic processes taken into account for the cooling rate. In particular, the $T$ sequence in region II can be easily changed by the elemental abundance, atomic levels and recombination processes (both radiative and dielectronic) and so forth, because bound electrons of heavy elements still remain to play an important role for radiation processes at temperatures lower than $100 \mathrm{eV}$, a distinct leap in region III.

9. The presence of hydrostatic gas surrounding a compact object may be related to the warm absorbers of AGNs. The ionization structure and the radiation spectrum investigated in the present work will be helpful to understand the spectrum formation and the interpretation of the absorption lines/edges observed.

Acknowledgements. The authors would like to thank the referee for his/her interesting comments and discussion, particularly about the instability. This work has been supported by the Grant in Aid (13640243) for Scientific Research of the Ministry of Education and Science in Japan. 


\section{References}

Allen, C. W. 1973, Astrophysical Quantities (The Athlone Press, University of London)

Hess, C. J., Kahn, S. M., \& Paerels, F. 1997, ApJ, 478, 94

Krolik, J. H., McKee, C. F., \& Tarter, C. B. 1981, ApJ, 249, 422

Krolik, J. H., \& Kriss, G. A. 1995, ApJ, 447, 512

Kallman, T. R., \& McCray, R. 1982, ApJS, 50, 263

Hatchett, S., Buff, J., \& McCray, R. 1982, ApJ, 206, 847

Hirano, T., Hayakawa, S., Nagase, F., Masai, K., \& Mitsuda, K. 1987, PASJ, 39, 619

Kawashima, K., \& Kitamoto, S. 1996, PASJ, 48, L113

Liedahl, D. A., \& Paerels, F. 1996, ApJ, 468, L33

Masai, K. 1984, Ap\&SS, 106, 391
Masai, K., Hayakawa, S., \& Nagase, F. 1988, J. Phys. Colloq. France, 49, C1-47

Masai, K. 1994, ApJ, 437, 770

Nayakshin, S., Kazanas, D., \& Kallman, T. R. 2000, ApJ, 537, 833

Porquet, D., \& Dubau, J. 2000, A\&AS, 143, 495

Raymond, J. C. 1993, ApJ, 412, 267

Reynolds, C. S., \& Fabian, A. C. 1995, MNRAS, 273, 1167

Sako, M., Liedahl, D. A., Kahn, S. M., \& Paerels, F. 2000, ApJ, 525, 921

Tarter, C. B., Tucker, W. H., \& Salpeter, E. E. 1969, ApJ, 156, 943

Woods, D. T., Klein, R. I., Castor, J. I., \& McKee, C. F. 1996, ApJ, 461, 767 